\title{
Readmission After Pancreaticoduodenectomy: An Issue of Survival and Survivorship
}

\author{
Geoffrey Porter, MD, FRCSC, FACS \\ Department of Surgery, Dalhousie University and QEII Health Sciences Centre, Halifax, Nova Scotia, Canada B3H 2Y9
}

I vividly remember an experience in the surgeons' lounge a few years ago where a junior colleague had just completed a difficult pancreaticoduodenectomy (PD) in a 72-year-old man with pancreatic cancer. A senior, somewhat crusty surgeon who was following his junior colleague in the operating room and was now 2 hours late, remarked loudly: "The only positive consequence of that operation is that I will likely miss dinner with my daughter's in-laws."

Long-term survival in pancreatic cancer remains an uncommon occurrence; this is attributable to the advanced stage at presentation, accelerated natural history, and the at best modest success of systemic therapy. Despite this, survival is the primary (if not singular) goal after PD for resectable pancreatic cancer. On the other hand, survivorship, defined as "the physical, psychosocial, and economic issues of cancer, from diagnosis until the end of life" by the National Cancer Institute, has been recognized as an increasingly important aspect of clinical cancer research. ${ }^{1}$ The study by Yermilov et al. examining readmission to hospital after PD for pancreatic cancer deals with both concepts. $^{2}$

Yermilov et al. provide a population-based analysis of readmission to hospital within a year for patients with pancreatic cancer undergoing PD in the state of California over a 10 -year period. The $59 \%$ one-year readmission rate identified by these investigators informs us that this event, inherently considered to be negative, will occur in most patients. Accepting, as acknowledged by the authors, the limitations of the use of administrative databases in the examination of clinical issues, it seems that these

(C) Society of Surgical Oncology 2008

First Received: 1 October 2008;

Published Online: 31 December 2008

G. Porter, MD, FRCSC, FACS

e-mail: Geoff.Porter@dal.ca readmissions can be broadly categorized into two groups: patients with perioperative morbidity as a consequence of PD, and patients with progressive disease-related symptomatology. In very few surgically treated malignancies are we left with these two distinct clinical scenarios occurring so commonly, and with relatively equal frequency, within a year of operation. It may be useful to think of these two issues separately in contemplating strategies to reduce such readmissions.

\section{POSTOPERATIVE MORBIDITY}

Surgical technique in PD and its potential impact on perioperative morbidity has been reasonably well studied over the past 15 years. ${ }^{3}$ Randomized clinical trials have been performed examining the roles of pyloric preservation (no difference), extended lymphadenectomy (increased morbidity), postoperative erythromycin (decreased morbidity), and pancreatic anastomotic technique (no difference) in PD. Several well-conducted cohort studies have examined the impact on post-PD morbidity of preoperative biliary drainage (unclear effect, multicenter randomized trial ongoing) and portal vein resection (no increase). Important volume outcome relationships have been identified with higher volume institutions and/or surgeons demonstrating improved perioperative mortality and lower length of stay (surrogate for perioperative morbidity). ${ }^{4}$ Even more importantly, regionalization of PD has been shown to be associated with improvements in postoperative mortality ${ }^{5}$; this may also apply to postoperative morbidity.

Given these and other important knowledge gains, how can readmissions for PD-related morbidity be reduced? Yermilov et al. suggest that better prevention strategies and use of home health services may prevent some readmissions, specifically those related to dehydration, deep-vein thrombosis/pulmonary embolus, and gastritis/upper 
gastrointestinal bleeding. Although one could conceive that specific strategies aimed at preventing such outcomes, or treating patients as outpatients, may reduce readmissions, there is little evidence to support this. A recent metaanalysis demonstrated that strong data to suggest that interventions may have a positive impact on readmissions does not exist. ${ }^{6}$ The few successful existing interventions tend to involve educational and resource components and combine predischarge and postdischarge interventions. It is possible that from a postoperative morbidity standpoint after PD, the incremental gains yet to be made are small.

\section{PROGRESSIVE PANCREATIC CANCER SYMPTOMATOLOGY}

Survivorship is a subject of increased emphasis in patients undergoing cancer treatment; this is predominantly based on the increasing number of patients surviving for longer periods of time (e.g., breast cancer). Unfortunately, overall survival in pancreatic cancer has not changed appreciably over the past 30 years, and population-based data of survival after PD for pancreatic cancer has demonstrated minimal gains. ${ }^{7}$ Despite the aforementioned progress in refining surgical technique, as well as major efforts to define optimal adjuvant/neoadjuvant therapy approaches, it is probably best to consider localized pancreatic cancer as most commonly a systemic disease with less visible burden of disease. ${ }^{8}$ Even in accepting this reality, however, both the patient and the surgeon would consider readmission to hospital within a year of PD for progression of disease a failure. This failure, and its consequent impact on survivorship, is predominantly an issue of patient selection.

Appropriate and optimal patient selection in pancreatic cancer can be considered at several levels, including screening and early detection, accurate and reproducible preoperative radiographic assessment, surgical treatment, use of adjuvant/neoadjuvant approaches, and surveillance. As with postoperative morbidity, better long-term outcomes are found in PD patients undergoing operation at high volume institutions; this has been, at least in part, attributed to selection factors. Critics of PD for pancreatic cancer in the past have accused surgeons of selecting the "good actors" for resection, thus reporting "artificially inflated" survival. We should embrace this approach at a population level, seek the "good actors," and provide optimal treatment. ${ }^{9}$

Yermilov et al. have made an important contribution in the surgical treatment of pancreatic cancer. They have shown that readmission within a year of PD (1) is common; (2) is underestimated by single-institution studies; and (3) often occurs $>3$ months after PD and is attributable to progressive disease. This work clearly illustrates the key dichotomy of PD for pancreatic cancer-clinically important perioperative morbidity and high death rate-and reinforces the importance of patient selection. We can use optimal patient selection to respond to the crusty senior surgeon, but more importantly, pancreatic cancer patients require it.

\section{REFERENCES}

1. National Cancer Institute, survivorship, in Dictionary of Cancer Terms. Available at: http://www.nci.nih.gov/Templates/db_alpha. aspx?CdrID=445089. Accessed October 21, 2008.

2. Yermilov I, Bentram, Sikeris E, et al. Readmissions following pancreaticoduodenectomy for pancreas cancer: a population-based appraisal. Ann Surg Oncol. (in press).

3. Ujiki MB, Talamonti MS. Guidelines for the surgical management of pancreatic adenocarcinoma. Semin Oncol. 2007;34:311-20.

4. Gouma DJ, van Geenen RC, van Gulik TM, et al. Rates of complications and death after pancreaticoduodenectomy: risk factors and the impact of hospital volume: toward the end of completion pancreatectomy. Ann Surg. 2000;232:786-95.

5. Langer B. Role of volume outcome data in assuring quality in HPB surgery. HPB (Oxford). 2007;9:330-4.

6. Mistiaen P, Francke AL, Poot E, et al. Interventions aimed at reducing problems in adult patients discharged from hospital to home: a systematic meta-review. BMC Health Serv Res. 2007;7:47.

7. Ries LAG, Melbert D, Krapcho M, et al, editors. SEER Cancer Statistics Review, 1975-2005. Bethesda, MD: National Cancer Institute, 2008. Available at: http://seer.cancer.gov/csr/1975_2005/. Accessed October 21, 2008.

8. Merchant N, Berlin J. Past and future of pancreas cancer: are we ready to move forward together? J Clin Oncol. 2008;26:3478-80.

9. Birkmeyer JD, Sun Y, Wong SL, et al. Hospital volume and late survival after cancer surgery. Ann Surg. 2007;245:777-83. 